\title{
Modellgestützte Bestimmung der komplexen Permittivität heterogener Katalysatoren im instationären Fall
}

Dipl.-Ing. Thomas Jungkunz, Prof. Dr.-Ing. Gerhard Fischerauer Universität Bayreuth, Lehrstuhl für Mess- und Regeltechnik, Universitätsstraße 30, 95447 Bayreuth 0921 55-7232 (Telefon), 0921 55-7235 (Fax), mrt@uni-bayreuth.de

\section{Kurzfassung}

Die komplexe Permittivität $\underline{\varepsilon}=\varepsilon_{0}\left(\varepsilon_{\mathrm{r}}+\mathrm{j} \sigma / \omega \varepsilon_{0}\right)$ von Dielektrika kann bekanntermaßen dadurch bestimmt werden, dass man eine kleine Materialprobe in einen zuvor leeren Hohlraumresonator einbringt und aus den Änderungen der Resonanzfrequenz und -güte die relative Permittivität $\varepsilon_{r}$ und die elektrische Leitfähigkeit $\sigma$ bei der Kreisfrequenz $\omega$ bestimmt. Man unterstellt dabei, dass die Probe den Resonator nur in geringem Maße stört (Störungsrechnung) und insbesondere die Resonatorgüte groß bleibt [1]. Diese Annahme gilt nicht mehr uneingeschränkt, wenn die zu untersuchende Materialprobe sehr groß oder stark verlustbehaftet ist. Im vorliegenden Beitrag wird ein Modell vorgestellt, das die Verluste eines teilgefüllten kreiszylindrischen Hohlraumresonators a priori beinhaltet und daher auch im Falle großer Verluste (kleiner Resonatorgüten) gültig bleibt. Damit lässt sich die komplexe Permittivität einer großen, verlustbehafteten Materialprobe über Hochfrequenz-(HF-)Messungen bestimmen. Zudem kann man Änderungen in den Materialeigenschaften infolge von beispielsweise chemischen Reaktionen in der Materialprobe (wie in Katalysatoren) oder infolge von Stoffanlagerungen (wie in Partikelfiltern) in situ ermitteln.

\section{Einleitung}

Ein Beispiel für einen praktisch wichtigen heterogenen Katalysator im instationären Betrieb ist der DreiWege-Katalysator (TWC) aus der automobilen Abgasnachbehandlung. Ein solcher TWC besteht aus einem keramischen Wabenkörper, ist mit einem Washcoat überzogen und in einem Metallgehäuse eingefasst. Die Bestandteile des Washcoats erfüllen unterschiedliche Aufgaben mit dem Ziel, Schadstoffe effizient aus dem Abgas zu eliminieren. Aluminiumoxid sorgt für eine große Oberfläche, Platin und andere Edelmetalle dienen als katalytisch aktive Komponenten und Ceroxid gleicht als Sauerstoffspeichermaterial Schwankungen im Sauerstoffgehalt der Verbrennungsabgase aus. Genaue Informationen über die Sauerstoffbeladung des Katalysators sind entscheidend für eine emissionsoptimierte Motorsteuerung, weil damit der Durchbruch von Schadgasen rechtzeitig verhindert werden kann [2, 3, 4].

Das katalysatorgefüllte Gehäuse ist aus hochfrequenztechnischer Sicht nichts anderes als ein teilgefülter kreiszylindrischer Hohlraumresonator (Bild 1). Der Oxidationsgrad des Katalysators lässt sich direkt, in situ und berührungslos beobachten, indem man die Frequenzlage und Güte der Hohlraumresonanzen vermisst. Denn die komplexe Permittivität von Ceroxid ändert sich, je nachdem ob es als $\mathrm{CeO}_{2}$ vorliegt (bei $\mathrm{O}_{2}$-Überschuss) oder als $\mathrm{Ce}_{2} \mathrm{O}_{3}$ (bei $\mathrm{O}_{2}$-Mangel) $[5,6,7]$.

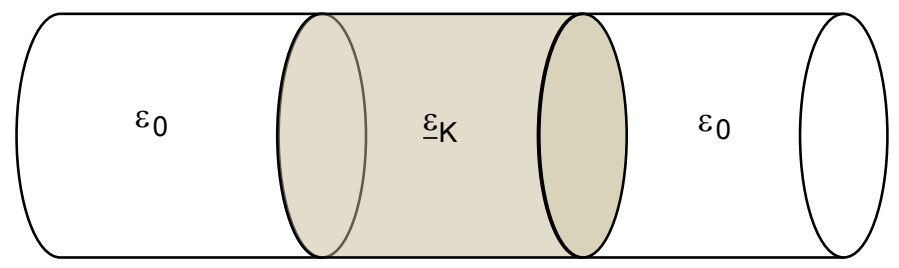

Bild 1: Katalysatorgefüllter Hohlraumresonator mit den Abschnitten Luft - Katalysator - Luft

Die Frequenz und Güte eines Resonanzmodus kann man z. B. aus den Streu-(S-)Parametern extrahieren, die mit einem vektoriellen Netzwerkanalysator (NWA) gemessen werden (Abschnitt 2). Die Lösung des inversen Problems, also die quantitative Bestimmung der Materialparameter des Katalysators aus den gemessenen S-Parametern, erfordert ein Modell, das auch bei großen Verlusten, sprich kleinen Güten, gültig bleibt. Das übliche Vorgehen, die Feldverteilung im Hohlraum für den verlustlosen Fall zu 
berechnen und die Auswirkung der Verluste als kleine Störung zu behandeln (wie es numerische Lösungsverfahren, etwa Finite-Elemente-Programme, in aller Regel tun), scheitert hier. Stattdessen sind die Verluste von vornherein zu berücksichtigen. Ein entsprechendes Modell wird in Abschnitt 3 vorgestellt. Dabei wird der eigentlich heterogene (poröse) Katalysator als homogenes Material mit komplexer Permittivität modelliert (übliche Homogenisierung wie in der Effektivmedientheorie [9]). Damit können die homogenisierten Materialparameter eines Katalysators sowohl im längshomogenen (wie in Bild 1) als auch - durch Zerlegung in dünne homogene Scheibchen - im längsinhomogenen, transienten Zustand bestimmt werden (Abschnitte 4 und 5).

\section{S-Parametermessung eines katalysatorgefüllten Hohlraumresonators}

In Bild 2 ist der Messaufbau skizziert, wie er für $S$-Parametermessungen von katalysatorgefüllten Hohlraumresonatoren verwendet wird [2, 10, 11, 12]. Der Katalysator liegt symmetrisch eingebettet in einem leitfähigen, kreiszylindrischen Gehäuse, an dessen Enden Drahtgitter angebracht sind, um einen gut definierten elektromagnetischen Resonanzraum zu erzeugen. Die Anordnung wird vom Abgas durchströmt.

Zur Anregung und Beobachtung elektromagnetischer Felder im Hohlraumresonator wird ein NWA verwendet, der über Koaxialkabel und kapazitive Stiftkoppler angekoppelt wird. Der NWA misst die frequenzabhängigen S-Parameter des Hohlraumresonators (s. Bild 3 für ein Beispiel) [8, S. 120]. Im Beispiel des TWC hat eine Reduktion von $\mathrm{CeO}_{2} \mathrm{zu} \mathrm{Ce}_{2} \mathrm{O}_{3}$ zur Folge, dass sowohl die Resonanzfrequenz als auch die Resonanzgüte sinken (Verschiebung der Resonanzkurve zu niedrigeren Frequenzen hin bei gleichzeitiger Verbreiterung der Kurve). Der Grund dafür liegt in steigenden Verlusten im Katalysator, welche mit der Änderung der komplexen Permittivität von Ceroxid einhergehen.

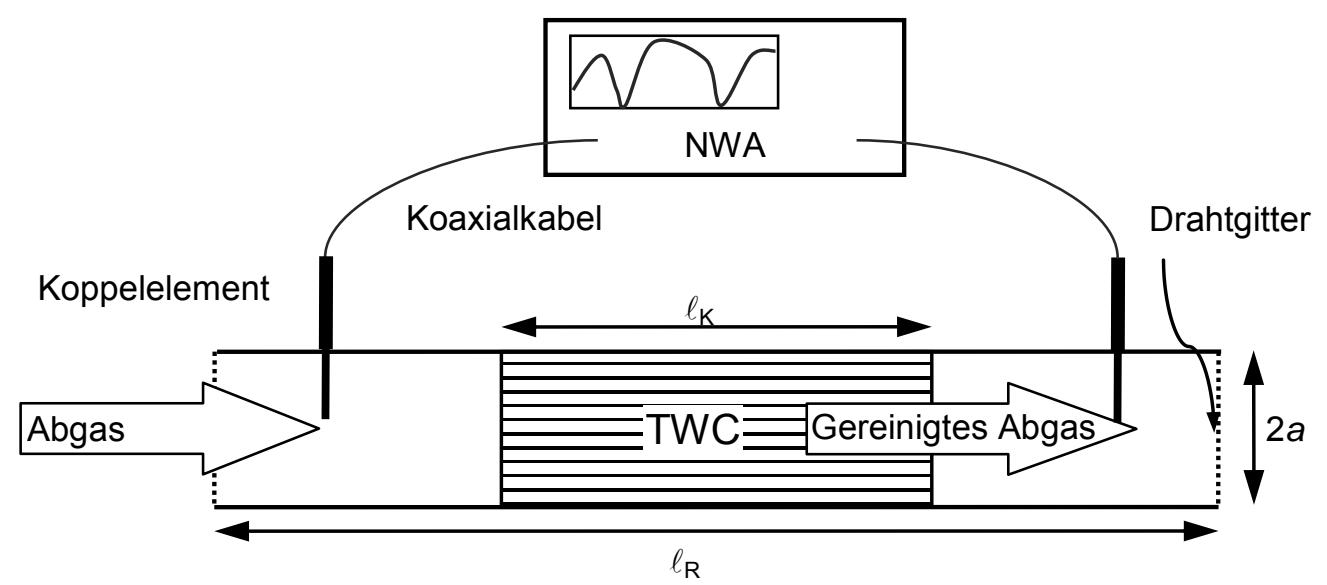

Bild 2: Schematischer Aufbau zur Messung der S-Parameter von kreiszylindrischen Hohlraumresonatoren des Radius a und der Länge $\ell_{R}$, mit symmetrisch eingebautem Katalysator der Länge $\ell_{K}$.

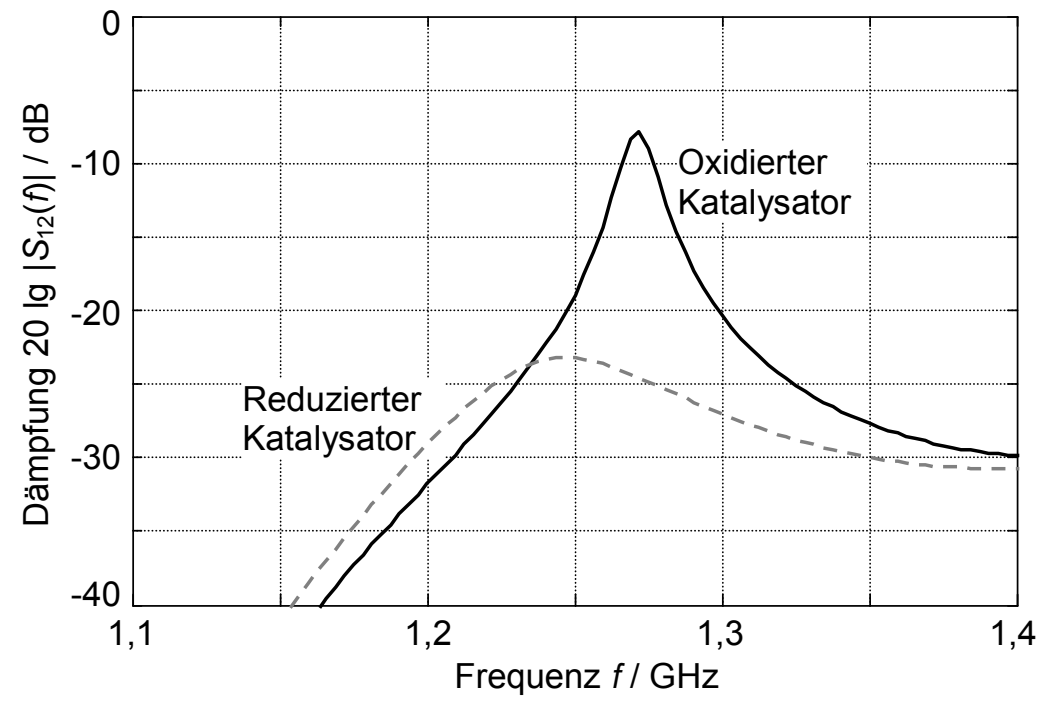

Bild 3: Betragsgang des Transmissionsfaktors eines mit zwei kapazitiven Stiftkopplern angekoppelten, gehäusten TWC. 
Beim Wechsel zwischen oxidierend und reduzierend wirkendem (sauerstoffreichem und sauerstoffarmem) Abgas ändert sich der Oxidationsgrad der elektrochemisch wirksamen Komponente eines TWC. Nach einem Wechsel läuft eine Reaktionsfront durch den Katalysator, so dass dieser axial nicht mehr homogen beladen ist. Eine Luftzahl-(Lambda-)Sonde nach dem Katalysator „merkt“ einen Wechsel der Abgaszusammensetzung daher erst, wenn die Reaktionsfront durch den Katalysator gelaufen ist, wenn also der Katalysator entweder voll mit Sauerstoff beladen oder voll entladen wurde (Bild 4a; $\lambda>1$ und

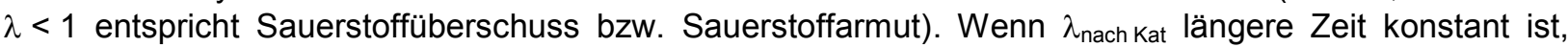
kann die (hier: Sauerstoff-)Beladung des Katalysators als homogen angenommen werden.

Diese Zustandsänderungen spiegeln sich auch in den Resonanzfrequenzen und -güten des katalysatorgefüllten Hohlraumes wider. Beide Größen bleiben konstant, wenn der Katalysator elektrochemisch stationär ist, und ändern sich mit der Zeit, wenn eine Reaktionsfront durch den Katalysator läuft (Bild 4b).

a)
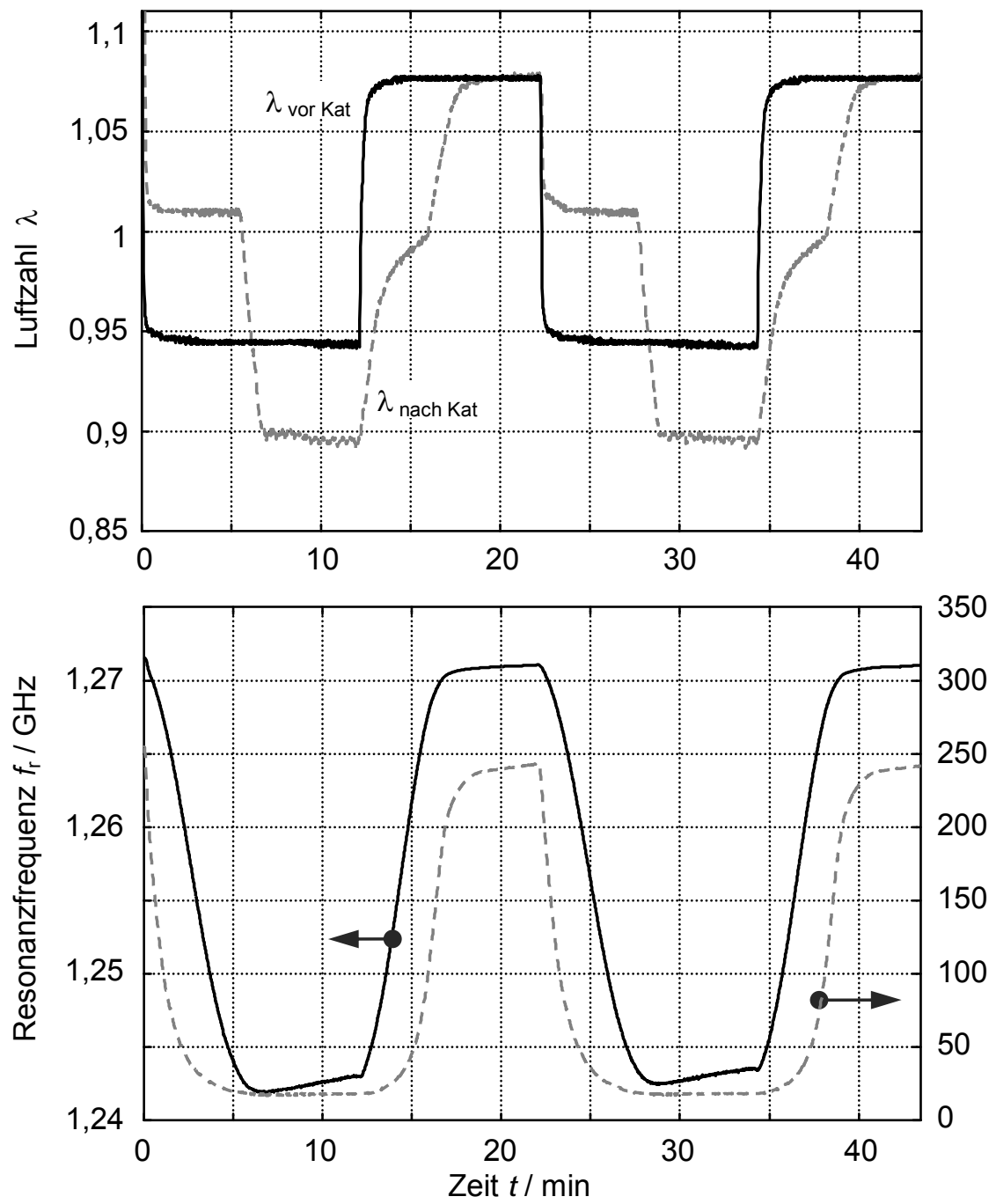

350

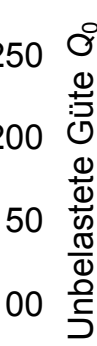

b)

Zeit $t / \min$

Bild 4: Reaktion eines gehäusten TWC auf periodisch wechselnde Abgaszusammensetzung (Messung). $Q_{v}=20 \mathrm{l} / \mathrm{min} ; T=400^{\circ} \mathrm{C}$. Trägergas $\mathrm{N}_{2}$ mit $10 \% \mathrm{CO}_{2}$ und $10 \% \mathrm{H}_{2} \mathrm{O}$. Für $\lambda>1$ wurden $1,2 \% \mathrm{O}_{2}$ und $550 \mathrm{ppm} N \mathrm{NO}$ zugegeben, für $\lambda<1$ dagegen $6000 \mathrm{ppm} \mathrm{CO}, 0,4 \% \mathrm{H}_{2}, 400 \mathrm{ppm} \mathrm{C}_{3} \mathrm{H}_{8}$ und $0,3 \% \mathrm{O}_{2}$. a) Luftzahl $\lambda$ vor und nach Katalysator. b) Resonanzfrequenz $f_{r}$ und unbelastete Güte $Q_{0}$ des TE $111-G_{\text {Gund- }}$ modus im TWC-gefüllten Hohlraum, bestimmt aus dem sekündlich gemessenen Transmissionsfaktor $\mathrm{S}_{12}$ (Messung mit NWA Rhode \& Schwarz ZVRE). Der Einfluss der Wandstromverluste auf die Güte wurde herausgerechnet, so dass $Q_{0}$ ausschließlich von den Verlusten im TWC rührt. 


\section{Modellierung eines axial inhomogenen kreiszylindrischen Hohlraumresonators}

Wir betrachten einen längsinhomogenen kreiszylindrischen Hohlraumresonator der Gesamtlänge $\ell_{R}$ und des Radius a. Zur Berücksichtigung der axialen Inhomogenität wird die Anordnung in $N$ Abschnitte der Länge $\ell_{i}$ mit jeweils konstanter Permittivität $\underline{\varepsilon}_{i}=\varepsilon_{r i} \varepsilon_{0}-\mathrm{j} \sigma_{i} / \omega$ unterteilt $(i=1, \ldots N$; Bild 5). Richtet man ein Zylinderkoordinatensystem $(\rho, \varphi, z)$ aus, wie in Bild 5 dargestellt, dann liegen die Grenzflächen des $i-$ ten Abschnitts bei $z_{i-1}$ und $z_{i}=z_{i-1}+\ell_{i}\left(z_{0}=0, z_{N}=\ell_{\mathrm{R}}\right)$.

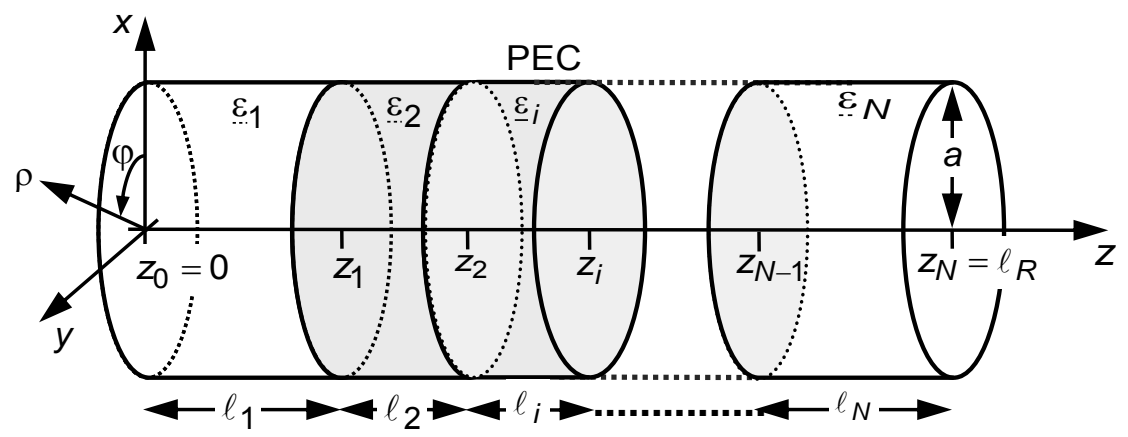

Bild 5: Modell eines kreiszylindrischen Hohlraumresonators mit elektrisch ideal leitenden Wänden (PEC). Längsinhomogene Materialeigenschaften werden mit $N$ dünnen Abschnitten homogener Permittivität modelliert.

Die Feldverteilung der transversal elektrischen (TE-)Moden, auf die wir uns hier beschränken, lässt sich aus den Modenfunktionen

$$
\Psi_{i, n m}=J_{n}\left(k_{\rho} \rho\right) \cdot \sin (n \varphi) \cdot\left(A_{i} \sin \left(k_{z i} z\right)+B_{i} \cos \left(k_{z i} z\right)\right)
$$

herleiten [13, S. 206]. Dabei bezeichnet $J_{n}(x)$ die Bessel-Funktion 1. Art und $n$-ter Ordnung, deren Ableitung $J_{n}^{\prime}(x)$ die Nullstellen $\xi_{n m}^{\prime}$ besitzt. Außerdem gilt

$$
\begin{aligned}
& k_{\rho}=k_{\rho, n m}=\frac{\xi_{n m}^{\prime}}{a} \text { und } \\
& k_{z i}^{2}=k_{z i, n m}^{2}=\omega^{2} \underline{\varepsilon}_{i} \mu_{0}-k_{\rho, n m}^{2}
\end{aligned}
$$

Die Modenfunktionen in GI. (1) sind so gewählt, dass die daraus bestimmte elektrische und magnetische Feldstärke den Maxwellschen Gleichungen genügt und die Randbedingungen bei $\rho=a$ erfüllt. Die Randbzw. Stetigkeitsbedingungen an den Grenzflächen $z=z_{i}$ werden durch geeignete Wahl der Amplituden $A_{i}$ und $B_{i}$ erfüllt. Das notwendige Verschwinden der tangentialen E-Feldkomponenten an den Enden des Resonators erzwingt $B_{1}=B_{N}=0$. Die Stetigkeit der Feldkomponenten an den anderen Grenzflächen erfordert weiter

$$
\begin{aligned}
& \Psi_{i, n m}\left(\rho, \varphi, z_{i}\right) \stackrel{!}{=} \Psi_{i+1, n m}\left(\rho, \varphi, z_{i}\right) \text { und } \\
& \frac{\partial \Psi_{i, n m}\left(\rho, \varphi, z_{i}\right)}{\partial z}=\frac{\partial \Psi_{i+1, n m}\left(\rho, \varphi, z_{i}\right)}{\partial z} .
\end{aligned}
$$

Die Zusammenfassung aller Randbedingungen führt auf ein homogenes lineares Gleichungssystem der Form $\boldsymbol{M}(\omega) \vec{x}=\overrightarrow{0}$ mit $\vec{x}=\left(A_{1}, A_{2}, B_{2}, \ldots, A_{N-1}, B_{N-1}, B_{N}\right)^{\top}$. Es hat nur dann eine nicht-triviale Lösung, wenn die Determinante $\operatorname{det} \boldsymbol{M}(\omega)$ verschwindet. Die mit dem ganzzahligen Index $p$ geordneten Lösungen $\underline{\omega}_{n m p}$ der Eigenwertgleichung $\operatorname{det} \boldsymbol{M}(\omega)=0$, die numerisch bestimmt werden müssen, sind komplexwertig, da nach $\mathrm{Gl}$. (3) die longitudinalen Wellenzahlen $k_{z i}$ und damit auch die Systemmatrix $\boldsymbol{M}$ komplex sind. Solche komplexen Kreisfrequenzen sind in folgendem Sinne zu interpretieren [14]:

$$
\underline{\omega}_{n m p}=\omega_{r, n m p}\left(1-\frac{j}{2 Q_{0, n m p}}\right)
$$


mit der Resonanzkreisfrequenz $\omega_{\mathrm{r}, n m p}=2 \pi f_{r, n m p}$ ( $f_{\mathrm{r}, n m p}$ ist die Resonanzfrequenz) und der unbelasteten Güte $Q_{0, n m p}$ des $\mathrm{TE}_{n m p}-$ Modus.

Bei gegebener Resonatorgeometrie hängen die Parameter $f_{\mathrm{r}}$ und $Q_{0}$ eines Modus nur von den komplexen Permittivitäten $\underline{\varepsilon}_{i}$ der $N$ Abschnitte ab. Zur Lösung des inversen Problems, wenn also von den gemessenen Merkmalen $f_{\mathrm{r}}$ und $Q_{0}$ auf die Materialparameter $\varepsilon_{\mathrm{r} i}$ und $\sigma_{i}$ geschlossen werden soll, werden die Materialparameter in der Simulation solange variiert, bis die berechneten Resonatoreigenschaften mit den gemessenen übereinstimmen. Bei der Lösung dieses Optimierungsproblems hat man die erlaubten Parameterwerte durch physikalische Überlegungen vorzugeben. So kann in einem TWC-Abschnitt die Leitfähigkeit nur zwischen der des voll oxidierten bzw. reduzierten TWC liegen.

\section{Materialparameteridentifikation im homogenen, stationären Zustand}

Ein Hohlraumresonator mit homogenem Katalysator nach Bild 1 umfasst drei homogene Abschnitte $(N=3)$. Bei symmetrischem Katalysatoreinbau gilt $\ell_{1}=\ell_{3}=\frac{1}{2}\left(\ell_{\mathrm{R}}-\ell_{\mathrm{K}}\right), \ell_{2}=\ell_{\mathrm{K}}$ und $\underline{\varepsilon}_{1}=\underline{\varepsilon}_{3}=\varepsilon_{0}$. In diesem Fall bleibt als einzig freier Parameter in der Simulation die Permittivität $\underline{\varepsilon}_{2}=\underline{\varepsilon}_{K}$ des Katalysators. Sie kann durch Rechnungs-Messungs-Anpassung bestimmt werden.

Bild 6a, b zeigt den Einfluss der relativen Permittivität $\varepsilon_{\mathrm{r}}$ und der Leitfähigkeit $\sigma$ des TWC, der dem Messergebnis von Bild $4 \mathrm{~b}$ zugrundelag $\left(\ell_{\mathrm{R}}=365 \mathrm{~mm}, \ell_{\mathrm{K}}=127 \mathrm{~mm}, a=62,5 \mathrm{~mm}\right)$, auf die Resonanz-

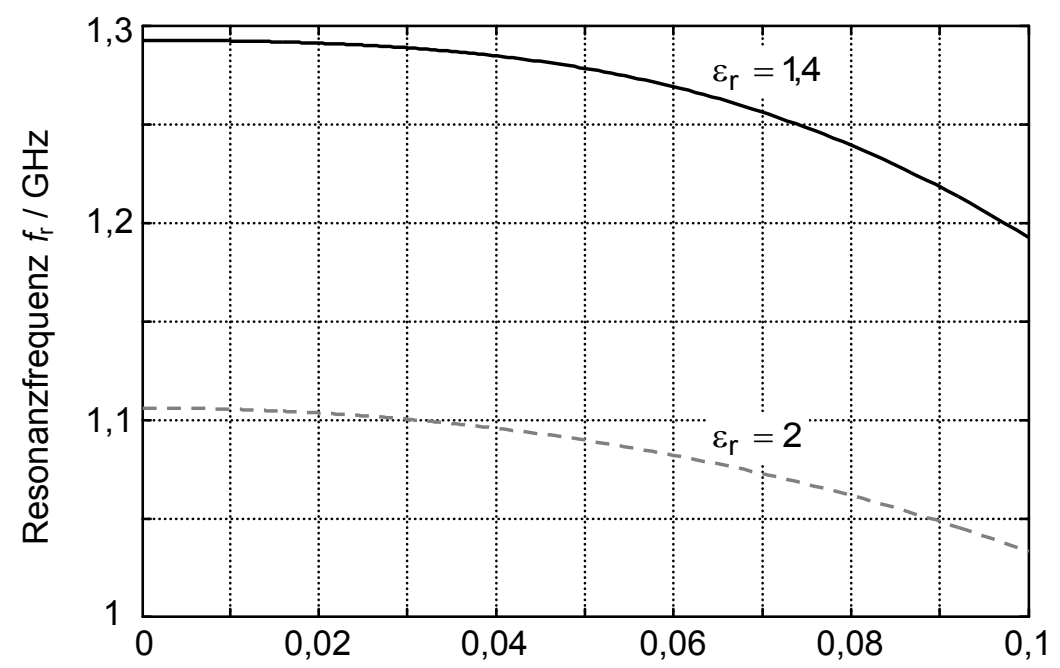

a)
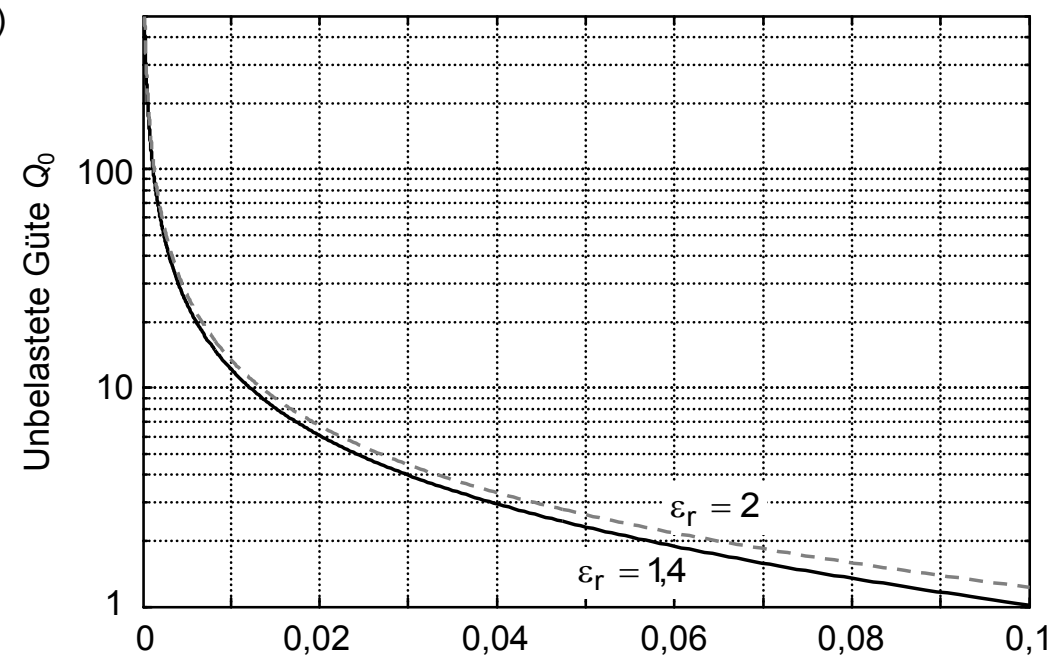

b)

Elektrische Leitfähigkeit $\sigma_{2} /(\mathrm{S} / \mathrm{m})$

Bild 6: Berechneter Einfluss der TWC-Materialeigenschaften auf a) die Resonanzfrequenz $f_{r}$ und b) die unbelastete Güte $Q_{0}$ des $\mathrm{TE}_{111}$-Modus in der Geometrie von Bild $1 \mathrm{bzw}$. hinter Bild 4 (siehe Text). 
Tabelle 1: Eigenschaften des $\mathrm{TE}_{111}$-Modus des gehäusten Katalysators, der der Messung von Bild 4 zugrunde lag, und daraus ermittelte Materialparameter des Katalysators.

\begin{tabular}{lccccc}
\hline Katalysatorzustand & \multicolumn{2}{c}{$\begin{array}{l}\text { Gemessene Resonanz- } \\
\text { parameter }\left(\mathrm{TE}_{111}\right), \text { Bild } 4 \mathrm{~b}\end{array}$} & & \multicolumn{3}{c}{$\begin{array}{l}\text { Geschätzte Materialparameter } \\
\text { des Katalysators }\end{array}$} \\
\cline { 2 - 3 } \cline { 5 - 6 } & $f_{\mathrm{r}} / \mathrm{GHz}$ & $Q_{0}$ & & $\varepsilon_{\mathrm{r}}$ & $\sigma /\left(10^{-4} \mathrm{~S} / \mathrm{m}\right)$ \\
\hline Homogen oxidiert & 1,271 & 250 & & 1,46 & 5 \\
\hline Homogen reduziert & 1,243 & 18 & & 1,54 & 68 \\
\hline
\end{tabular}

frequenz und die unbelastete Güte des $\mathrm{TE}_{111}$-Grundmodus. Für das Simulationsbeispiel wurde bei zwei konstanten Werten von $\varepsilon_{r}$ die Leitfähigkeit $\sigma$ von $10^{-4}$ auf $0,1 \mathrm{~S} / \mathrm{m}$ erhöht. Danach lassen sich für $\sigma<0,01 \mathrm{~S} / \mathrm{m}$ merkliche Änderungen der Resonanzfrequenz nur der Änderung von $\varepsilon_{\mathrm{r}}$ zuschreiben. Bei höheren Werten von $\sigma$ (größeren Verlusten) gilt das nicht mehr, denn hier sinkt die Resonanzfrequenz mit der Leitfähigkeit. Dagegen fällt die Güte im gesamten Leitfähigkeitsbereich stark mit der Leitfähigkeit.

Für die Beispielmessung aus Bild $4 \mathrm{~b}$ ergibt sich eine minimale Güte von circa 18 , was nach Bild $6 \mathrm{~b}$ auf $\sigma<0,01 \mathrm{~S} / \mathrm{m}$ schließen lässt. Die gemessene Änderung der Resonanzfrequenz in Bild $4 \mathrm{~b}$ geht daher hauptsächlich auf eine Änderung der relativen Permittivität im Katalysator zurück (mit Hilfe von Bild 6a schätzt man $a b$, dass $1,4 \leq \varepsilon_{r} \leq 1,6$ gelten muss).

Das Ergebnis der quantitativen Auswertung des Beispiels ist in Tabelle 1 aufgeführt. Wie vermutet, ändern sich danach sowohl die relative Permittivität als auch die elektrische Leitfähigkeit des TWC, was beides durch eine Strukturänderung von Ceroxid erklärt werden kann $[5,6,7]$.

\section{Schätzung ortsabhängiger Materialparameter im instationären Fall}

Bei transienten Vorgängen stellt sich in jedem Augenblick eine ortsabhängige Permittivität $\underline{\varepsilon}(z)$ in einem Katalysator ein. Dies wird gemäß Abschnitt 3 modelliert, indem der Katalysator in $N-2$ dünne Abschnitte der Permittivität $\underline{\varepsilon}_{i}=\varepsilon_{\mathrm{r} i} \varepsilon_{0}-\mathrm{j} \sigma_{i} / \omega$ zerlegt wird $(i=2, \ldots, N-1)$. Die $\varepsilon_{\mathrm{r} i}$ und $\sigma_{i}$ liegen zwischen den entsprechenden Werten des vollständig oxidierten oder reduzierten Katalysators. Beim TWC, der dem Messergebnis von Bild 4 zugrunde lag, liest man diese Grenzwerte in Tabelle $1 \mathrm{ab}$.

Die Momentanverteilung der Sauerstoffkonzentration im TWC - wir bleiben weiter bei diesem Beispiel und damit die Momentanverteilung von $\underline{\varepsilon}(z)$ hängen von den physiko-chemischen Details ab (Strömung, Sauerstoffdiffusion, chemische Reaktionen, ...) [15]. Näherungsweise kann man $\underline{\varepsilon}(z)$ durch eine Funktionenklasse $f(z ; \vec{p})$ mit einem Vektor freier Parameter $\vec{p}$ beschreiben. Der Parametervektor $\vec{p}$ ist dann so zu wählen, dass die Rechnung möglichst gut mit der Messung übereinstimmt.

Wir betrachten einen vollständig oxidierten TWC $\left(\varepsilon_{\mathrm{r}}(z)=\varepsilon_{\mathrm{rO}}=1,46\right.$ und $\sigma(z)=\sigma_{\mathrm{O}}=5 \cdot 10^{-4} \mathrm{~S} / \mathrm{m}$ laut Tabelle 1), der ab dem Zeitnullpunkt mit reduzierendem Abgas beaufschlagt wird. Die daraufhin durch den Katalysator wandernde Reaktion führt zu jedem Zeitpunkt $t$ zu einem monoton mit $z$ fallenden Verlauf sowohl von $\varepsilon_{\mathrm{r}}(z)$ als auch von $\sigma(z)$. Ähnlich dem Konzentrationsverlauf in einem idealen Strömungsrohr, in dem Reaktionen erster Ordnung ausschließlich mit Sauerstoff ablaufen und Reaktionspartner im Überschuss vorhanden sind, modellieren wir dies durch den wellenartig durch den TWC wandernden exponentiellen Verlauf

$$
\begin{aligned}
& \varepsilon_{\mathrm{r}}(\xi ; p)=\left\{\begin{array}{cc}
\varepsilon_{\mathrm{rO}}+\left(\varepsilon_{\mathrm{rR}}-\varepsilon_{\mathrm{rO}}\right) \cdot\left(1-\mathrm{e}^{p \xi}\right) & \text { für } \xi<0 \\
\varepsilon_{\mathrm{rO}} & \text { für } \xi>0
\end{array},\right. \\
& \sigma(\xi ; p)=\left\{\begin{array}{cc}
\sigma_{\mathrm{O}}+\left(\sigma_{\mathrm{R}}-\sigma_{\mathrm{O}}\right) \cdot\left(1-\mathrm{e}^{p \xi}\right) & \text { für } \xi<0 \\
\sigma_{\mathrm{O}} & \text { für } \xi>0
\end{array}\right.
\end{aligned}
$$

mit $\xi=z-z_{1}-v t\left(z_{1} \leq z \leq z_{2}, 0 \leq t\right.$; Bild 7a). Dabei ist $v$ die Geschwindigkeit der Reaktionsfront (aus Bild 4a liest man etwa ab: $v \approx 365 \mathrm{~mm} / 6 \mathrm{~min} \approx 1 \mathrm{~mm} / \mathrm{s}$ ). Weiterhin bezeichnen $\varepsilon_{\mathrm{rR}}=1,54$ und $\sigma_{\mathrm{R}}=$ $6,8 \cdot 10^{-3} \mathrm{~S} / \mathrm{m}$ die TWC-Eigenschaften im vollständig reduzierten Fall (vgl. Tabelle 1 ). 
a)
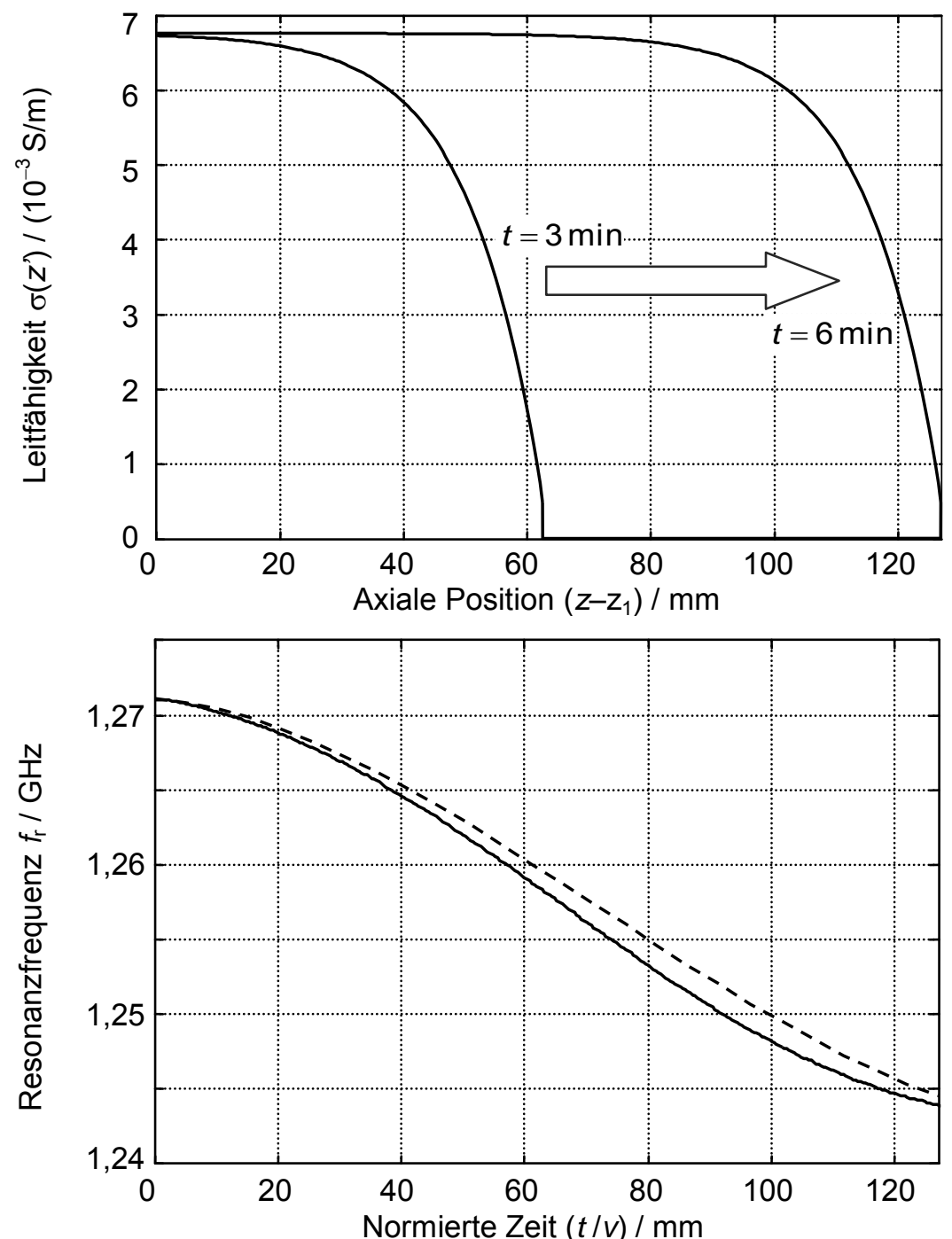

b)

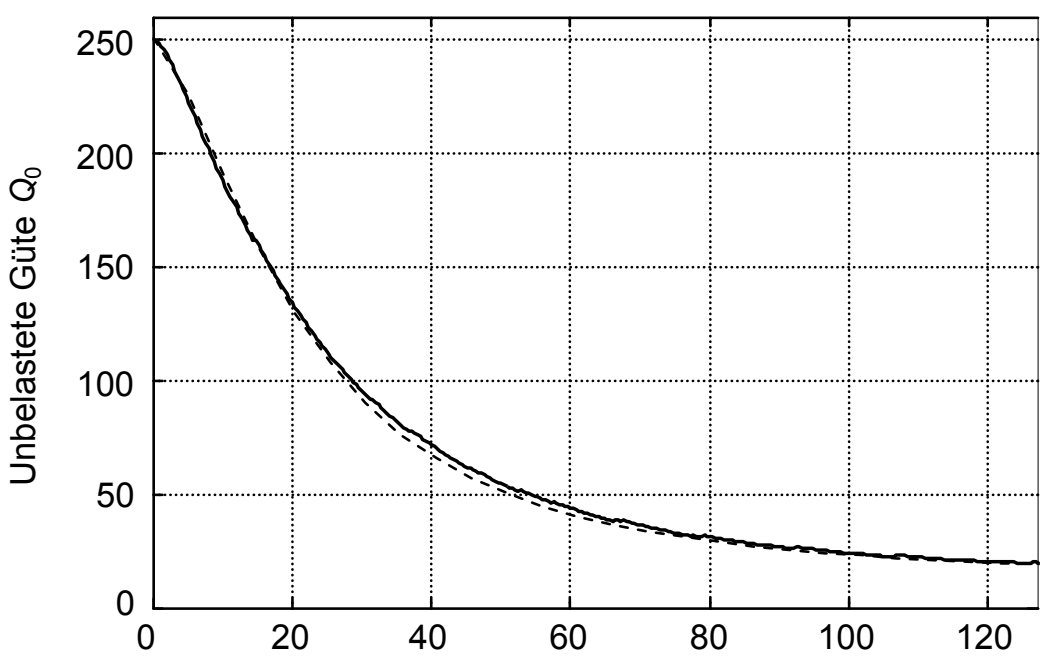

c)

Normierte Zeit $(t / v) / \mathrm{mm}$

Bild 7: TWC im instationären Betrieb, nachdem der zunächst vollständig oxidierte Katalysator schlagartig mit reduzierendem Abgas beströmt wurde. a) Angenommener Momentanverlauf der elektrischen Leitfähigkeit $\sigma$ entlang der Katalysatorachse für zwei Zeitpunkte nach dem Eintreffen des reduzierend wirkenden Abgases, berechnet nach GI. (8)mit $p=85$. b, c) Zeitabhängige Resonanzfrequenz und unbelastete Güte des $\mathrm{TE}_{111}$-Modus beim Wandern der Reaktionsfront durch den Katalysator (Geschwindigkeit $v \approx 1 \mathrm{~mm} / \mathrm{s}$ ). - - -: Simulation (Lösung der Eigenwertgleichung nach Abschnitt 3 zu jedem Zeitpunkt mit dem jeweiligen Momentanverlauf von $\sigma$ analog zu Bild a); ——: Messung (Bild 4b zwischen Minute 22 und Minute 28). 
Dass die einfache (nur durch einen Parameter $p$ parametrisierte) und in Details sicher verbesserungswürdige Modellierung der Reaktionsfront durch Gl. (7) und (8) dennoch die Verhältnisse sehr gut erfasst, wird durch den Rechnungs-Messungs-Vergleich in Bild 7b, c bestätigt. Der Durchlauf der in Bild 7a in zwei Momentaufnahmen dargestellten Materialparameterverteilung durch den Katalysator hat nämlich nach Abschnitt 3 einen Zeitverlauf der Resonanzfrequenz und der Resonanzgüte des $\mathrm{TE}_{111}$-Modus zur Folge, der sehr gut zum gemessenen Zeitverlauf passt. Alle wesentlichen Details des Experiments werden richtig beschrieben: geringere Anfangs- und Endsteigung der $f_{r}(t)$-Kurve, Wendepunkt in der $f_{r}(t)$ Kurve, in etwa hyperbolischer Verlauf der $Q_{0}(t)$-Kurve usw. Die noch zu beobachtende Abweichung zwischen der simulierten und der gemessenen $f_{r}(t)$-Kurve verwundert nicht, wenn man die einfachen Annahmen zum Verlauf der Reaktionsfront berücksichtigt. Ohne Zweifel würde eine realistischere Beschreibung der Orts- und Zeitabhängigkeit der Materialparameter eines Katalysators die Abweichung noch kleiner werden lassen.

Es hat sich also gezeigt, dass das beschriebene Verfahren die experimentell beobachteten Zeitverläufe der Resonanzparameter eines katalysatorgefüllten Gehäuses bei instationärem Betrieb sehr gut beschreibt. Im Umkehrschluss lässt sich die momentane Ortsabhängigkeit der Materialparameter eines Katalysators, z. B. also auch der Ort einer Reaktionsfront, schätzen, wenn man die Resonanzparameter kennt. Beim vorliegenden einfachen Modell würde man aus den gemessenen Werten von $f_{\mathrm{r}}$ und $Q_{0}$ auf die dazu passende Kurve aus der Schar der möglichen Kurven von $\sigma(z, t)$, von denen zwei in Bild 7a dargestellt sind, schließen.

\section{Zusammenfassung und Ausblick}

Vorgestellt wurde ein Modell eines längsinhomogenen kreiszylindrischen Hohlraumresonators, das auch bei beliebig großen Verlusten (beliebig großer Leitfähigkeit) der Hohlraumfüllung gilt. Die Inversion des Modells erlaubt es, die inhomogene Verteilung der elektrischen Leitfähigkeit und der relativen Permittivität eines großen Testobjekts entlang der Zylinderachse zu schätzen. Damit wird es auch möglich, transiente Vorgänge im Katalysator (instationärer Betrieb) zu untersuchen. Konkret sind dafür zunächst die Resonanzfrequenz $f_{r}$ und die unbelastete Güte $Q_{0}$ eines Resonanzmodus zu messen, was z. B. mit Hilfe von $S$-Parameter-Messungen erfolgen kann. Sodann hat man von diesen Parametern auf eine dazu passende Ortsabhängigkeit der Materialparameter zu schließen. Dies beinhaltet die Annahme einer orts- und zeitabhängigen Funktionenklasse $f\left(z^{\prime} ; p_{i}\right)$ für die Materialparameterverteilungen. Schon eine einfache Funktionenklasse mit nur einem freien Parameter führt zu sehr guten Ergebnissen und könnte praxistauglich sein. Für noch bessere Ergebnisse benötigt man Funktionen, die hinreichend genau die chemischen, thermodynamischen und strömungsmechanischen Vorgänge im Katalysator berücksichtigen.

\section{Danksagung}

Die in Bild 4 ausgewerteten Messdaten stammen von Herrn Dr.-Ing. Sebastian Reiß und Herrn Dipl.-Ing. Matthias Spörl, was hier dankbar anerkannt wird. Die Messungen fanden an der Synthesegasanlage des Lehrstuhls für Funktionsmaterialen der Universität Bayreuth statt.

\section{Literatur}

[1] S. H. Chao, "Measurements of microwave conductivity and dielectric constant by the cavity perturbation method and their errors", IEEE Trans. MTT, Vol. 33, No. 6, Juni 1985, S. 519-526.

[2] R. Moos et al., „TWC: lambda control and OBD without lambda probe - an initial approach“, SAE World Congress, Detroit, Paper No. 2008-01-0916, 14.-17. Apr. 2008.

[3] G. Fischerauer et al., "Catalyst State Observation via the Perturbation of a Microwave Cavity Resonator", Frequenz, Vol. 62, No. 7-8, Sept. 2008, S. 180-184.

[4] G. Fischerauer et al., „On the inverse problem associated with the observation of electrochemical processes by the RF cavity perturbation method", Proc. Sixth Int'l Multi-Conf. on Systems, Signals \& Devices (SSD'09), Djerba, 23.-26. März 2009, DOI: 10.1109/SSD.2009.4956751.

-, "Observation of electrochemical processes by the RF cavity perturbation method and associated inverse problem“, Trans. Systems, Signals \& Devices, Vol. 6, No. 4, Jan. 2012, S. 1-17.

[5] H. L. Tuller, A. S. Nowick, „Defect structure and electrical properties of nonstoichiometric $\mathrm{CeO}_{2}$ single crystals“, J. Electrochem. Soc., Vol. 126, No. 2, 1979, S. 209-217.

[6] V. Grosse et al., „Conductivity and dielectric properties of thin amorphous cerium dioxide films”, J. Phys. D: Appl. Phys., Vol. 40, No. 4, 2007, S. 1146-1149. 
[7] S. B. Ogale et al., „High-K Candidates for Use as the Gate Dielectric in Silicon Mosfets: Thin Films and Heterostructures for Oxide Electronic", in: Multifunctional Thin Film Series, S. 31-78. New York: Springer, 2005.

[8] L.-F. Chen, Microwave electronics: Measurement and materials characterization. Chichester: Wiley, 2004.

[9] P. S. Neelakanta (Hrsg.), Handbook of electromagnetic materials. Boca Raton: CRC Press, 1995.

[10] G. Fischerauer, M. Förster, R. Moos, „Sensing the Soot Load in Automotive Diesel Particulate Filters by Microwave Methods“, Meas. Sci. Tech., Vol. 21, No.3, März 2010, S. 2950-2959.

[11] P. Fremerey et al., „Determination of the $\mathrm{NO}_{x}$ loading of an automotive lean $\mathrm{NO}_{\mathrm{x}}$ trap by directly monitoring the electrical properties of the catalyst material itself“, Sensors, Vol. 11, No. 9, Sept. 2011, S. 8261-8280.

[12] S. Reiß et al., „Monitoring the ammonia loading of zeolite-based ammonia SCR catalysts by a microwave method", Chem. Eng. Technol., Vol. 34, No. 5, Mai 2011, S. 791-796.

[13] R. F. Harrington, Time-Harmonic Electromagetic Fields. New York: McGraw-Hill, 1961.

[14] O. Klein et al., „Microwave cavity perturbation technique: Part III: Applications“, Int. J. Infrared and Millimeter Waves, Vol. 14, No. 2, 1993, S. 2489-2517.

[15] N. Mladenov, Modellierung von Autoabgaskatalysatoren, Dissertation, Univ. Karlsruhe, 2009. 